\title{
Magnetic Field and Helicity in Solar Active Regions
}

\author{
Hongqi Zhang \\ National Astronomical Observatories, Chinese Academy of Sciences, \\ Beijing 100012,China, E-mail: hzhang@bao.ac.cn
}

The measurements of photospheric vector magnetograms provide an important chance to understand the basic properties of the solar magnetic field and the relationship with magnetic helicity in the solar atmosphere. Some questions on the analysis of photospheric vector magnetograms obtained at Huairou solar Observing Station have been discussed:

(1) The slight deviations for the data sets of vector magnetograms obtained at different observatories normally can be fond, which probably caused by the observing methods, magneto-optical effects and data reducing process etc. The analysis of magneto-optical effects on the measurements of vector magnetic field with $\mathrm{Fe} I \lambda 5324.19 \AA$ line by Huairou vector magnetograph (and the comparison with some observing results obtained other magnetographs) provides an estimation on the measurement accuracy of magnetic field.

(2) Several methods (such as, $\bar{\alpha}, \alpha_{\text {best }}$ or $\left.\mathbf{B} \cdot(\nabla \times \mathbf{B})_{\|}\right)$can be used to analyze the chirality of magnetic field inferred from photospheric vector magnetograms. Due to the incompleteness of the observational magnetic field data for the calculation of current helicity density etc in the solar atmosphere, some priori assumptions probably have been remained, which also cause some nondeterminacy on the analysis of helicity.

(3) Due to the opacity of solar photosphere, the generation of helical magnetic field in the solar sub-atmosphere is an interesting topic. The relationship between the newly emerging magnetic flux and evolution of the helicity in the solar atmosphere provides an important massage on the transfer of magnetic helicity from the sub-atmosphere into the solar atmosphere, which is also necessary for us to understand the possible geometry of magnetic field in the sub-atmosphere and the topology of helical magnetic field in the corona. A relevant result is that as the emergence of kinked magnetic ropes formed in the sub-atmosphere, one probably can detect the possible trace from a series of photospheric vector magnetograms. 